\title{
Two-lung ventilation in the prone position: is it the standard anesthetic management for thoracoscopic esophagectomy?
}

\author{
Hiroya Takeuchi · Yuko Kitagawa
}

Received: 25 November 2013/Published online: 4 February 2014

(C) The Japanese Association for Thoracic Surgery 2014

\begin{abstract}
Although esophagectomy with radical lymphadenectomy is one of the most invasive gastrointestinal surgeries, esophagectomy remains the mainstay of potentially curative treatment for patients with localized esophageal cancer. Therefore, esophagectomy via a thoracoscopic and/or laparoscopic approach seems to be very attractive as a less invasive surgery. Since 1992, when Cuschieri et al. [1] first reported on thoracoscopic esophagectomy as minimally invasive esophagectomy (MIE), technical advancements and the development of endoscopic equipment, which are available for esophageal resection as well as extended mediastinal lymphadenectomy, have resulted in increase in the popularity of MIE. However, the advantages with regard to short-term outcome and the oncological feasibility of MIE have not been adequately established [2].

Till date, two types of patient positions have been used for thoracoscopic esophagectomy. Most thoracic surgeons prefer performing right transthoracic thoracoscopic esophagectomy in the left lateral decubitus position, similar to right transthoracic open esophagectomy. However, since Cuschieri et al. [1] first described thoracoscopic mobilization of the esophagus in the prone position, thoracoscopic esophagectomy in the prone position has rapidly gained popularity [3-6].

The best advantage of the prone position is that a good surgical field and view of the posterior mediastinum, including the esophagus, can be obtained without any
\end{abstract}

This comment refers to the article available at doi:10.1007/s11748013-0335-0.

H. Takeuchi $(\bowtie) \cdot$ Y. Kitagawa

Department of Surgery, Keio University School of Medicine,

35 Shinanomachi, Shinjuku-ku, Tokyo 160-8582, Japan

e-mail: htakeuchi@a6.keio.jp retraction of the right lung using a retractor or sutures, because the right lung naturally falls away under gravity in the prone position and because of additional carbon dioxide insufflation of the thoracic cavity. Several studies have demonstrated that thoracoscopic esophagectomy in the prone position may result in a shorter operating time and lower incidence of postoperative respiratory complications compared with that in the left lateral decubitus position [3, 7]. However, the prone position is still considered to be problematic in terms of the safety of the procedure because, in this position, it is technically difficult to perform urgent conversion to right thoracotomy in an emergency situation such as sudden massive bleeding [8]. A comparison of the left lateral decubitus position with the prone position should be assessed using randomized controlled trials to determine the appropriate positioning for MIE [9].

Thoracoscopic esophagectomy has been essentially performed under one-lung ventilation, even in the prone position. However, a recent article by Saikawa et al. [10] reported on thoracoscopic esophagectomy under twolung ventilation in the prone position. They retrospectively evaluated the chronological changes in intraoperative respiration and hemodynamics in 14 patients who underwent thoracoscopic esophagectomy with two-lung ventilation in the prone position. As results, excessive increases in airway pressure or evident circulatory depressions were not observed with the addition of an artificial pneumothorax to the right thoracic cavity or a bilateral pneumothorax due to the injury of left mediastinal pleura during the thoracoscopic procedures. They concluded that the artificial pneumothorax under twolung ventilation may be beneficial for maintaining stable hemodynamics and oxygenation in thoracoscopic esophagectomy in the prone position. 
Although these findings should be interpreted with caution because the study had several limitations, they have provided a valuable reference for demonstrating the safety of thoracoscopic esophagectomy under two-lung ventilation in the prone position. In fact, the method with two-lung ventilation is assumed to have several advantages over that with conventional one-lung ventilation in terms of facilitating anesthesia induction and maintenance in the prone position, and favorable tracheal mobility when performing precise lymph node dissection along the left recurrent laryngeal nerve. If the safety of this procedure could be validated, two-lung ventilation would become a standard procedure in thoracoscopic esophagectomy in the prone position in the future.

However, the study by Saikawa et al. [10] seemed to have several issues to be resolved. In this study, the authors aimed to preserve the left mediastinal pleura for as long as possible during the thoracoscopic procedure to prevent the unexpected collapse of the left lung due to the bilateral artificial pneumothorax by opening the left mediastinal pleura. However, a significant change in respiratory status and/or hemodynamics may occur when the left mediastinal pleura is widely opened at an early phase during the thoracoscopic procedure. In addition, in case that require conversion to open thoracotomy, two-lung ventilation may interrupt urgent treatment with expanded right lung. Anyhow, as they had also described in their manuscript, the clinical utility and relevance of two-lung ventilation in the prone position should be evaluated in a prospective comparison study with conventional one-lung ventilation in terms of not only the intraoperative respiratory status and hemodynamics but also surgical parameters such as the operating time, blood loss, number of retrieved mediastinal lymph nodes, and postoperative morbidity. Moreover, the two approaches should be finally compared in terms of long-term survival.

Nevertheless, comparison studies are needed to confirm the superiority of the prone position over the left lateral decubitus position in MIE. Simultaneously, further exploration may precisely clarify whether two-lung ventilation in the prone position could revolutionize MIE for esophageal cancer.

Conflict of interest All the authors have declared no competing interest.

\section{References}

1. Cuschieri A, Shimi S, Banting S. Endoscopic esophagectomy through a right thoracoscopic approach. J R Coll Surg Edinb. 1992;37:7-11.

2. Takeuchi H, Kawakubo H, Kitagawa Y. Current status of minimally invasive esophagectomy for patients with esophageal cancer. Gen Thorac Cardiovasc Surg. 2013. (Epub ahead of print).

3. Palanivelu C, Prakash A, Senthilkumar R, Senthilnathan P, Parthasarahi R, Rajan PS, et al. Minimally invasive esophagectomy: thoracoscopic mobilization of the esophagus and mediastinal lymphadenectomy in prone position-experience of 130 patients. J Am Coll Surg. 2006;203:7-16.

4. Fabian T, McKelvey AA, Kent MS, Federico JA. Prone thoracoscopic esophageal mobilization for minimally invasive esophagectomy. Surg Endosc. 2007;21:1667-70.

5. Watanabe M, Baba Y, Nagai Y, Baba H. Minimally invasive esophagectomy for cancer: an updated review. Surg Today. 2013;43:237-44.

6. Ozawa S, Ito E, Kazuno A, et al. Thoracoscopic esophagectomy while in a prone position for esophageal cancer: a preceding anterior approach method. Surg Endosc. 2013;17:40-7.

7. Fabian T, Martin J, Katigbak M, McKelvey AA, Federico JA. Thoracoscopic esophageal mobilization during minimally invasive esophagectomy: a head-to head comparison of prone versus decubitus positions. Surg Endosc. 2008;22:2485-91.

8. Kaburagi T, Takeuchi H, Kawakubo H, Omori T, Ozawa S, Kitagawa Y. Clinical utility of a novel hybrid position combining the left lateral decubitus and prone positions during thoracoscopic esophagectomy. World J Surg. 2014;38:410-8.

9. Jarral OA, Purkayastha S, Athanasiou T, Darzi A, Hanna GB, Zacharakis E. Thoracoscopic esophagectomy in the prone position. Surg Endosc. 2012;26:2095-103.

10. Saikawa D, Okushiba S, Kawata M, Okubo T, Kitashiro S, Kawarada Y, Suzuki Y, Kato H. Efficacy and artificial pneumothorax under two-lung ventilation in thoracoscopic esophagectomy for esophageal cancer in the prone position. Gen Thorac Cardiovasc Surg. 2013. in press. 